\title{
Quantum Correlations Among Superradiant Bose-Einstein Condensate Atoms ${ }^{1}$
}

\author{
M. E. Taşgın ${ }^{a}$, B. Öztop ${ }^{a}$, M. Ö. Oktel ${ }^{a}$, and Ö. E. Müstecaplıõ̃lu ${ }^{b}$ \\ ${ }^{a}$ Department of Physics, Bilkent University, Bilkent, Ankara, 06800 Turkey \\ ${ }^{b}$ Department of Physics, Koç University, Sartyer, Istanbul, 34450 Turkey \\ e-mail:metasgin@fen.bilkent.edu.tr \\ Received August 3, 2009
}

\begin{abstract}
Quantum correlations among atoms in superradiant Bose-Einstein condensates are discussed. It is shown that atoms in the superradiant atomic condensate can exhibit continuous variable quantum entanglement analogous to Einstein-Podolsky-Rosen (EPR)-type quantum correlations. Comparison to quantum entanglement in the Dicke model in thermal equilibrium is provided.
\end{abstract}

DOI: $10.1134 / \mathrm{S} 0030400 \mathrm{X} 10030185$

\section{INTRODUCTION}

Quantum correlations and dramatic changes in the behavior of a quantum collective system depending on the strength of interactions among the constituents of the many body system show great parallelism [1]. Quantum phase transitions are associated with singularities in the ground state energy of many-body systems which occur at certain values of interaction parameters. Such singularities at critical points are usually accompanied by changes in the nature of quantum correlations. Particular emphasis was given to characterize quantum entanglement properties of quantum collective systems during quantum phase transitions. For that aim, generic models including spin, fermion and boson systems are discussed (for a recent review see Ref. [2] and references therein).

Most of the specific models that has been studied for quantum entanglement and quantum phase transitions are on interacting spin systems, such as LipkinMeshov-Glick Model [3, 4] or one-dimensional spin lattices [5-7]. Apart from such spin chain studies, a notable system that has been investigated is the so called Dicke model of single-mode superradiance [8]. Explorations of the entanglement properties of the Dicke model are so far restricted to thermal equilibrium and ground state entanglement between atoms and the radiation field [9]. In addition to that thermal equilibrium situation, superradiance can also be considered as a time-dependent effect in which superradiant pulses with maximum intensity proportional to the square of the number of atoms in the radiating sample are emitted.

A recent experimental system in which superradiant pulses are generated is the Bose-Einstein condensate [10]. Quantum entanglement between condesate

\footnotetext{
${ }^{1}$ The article is published in the original.
}

atoms and superradiant pulse photons was proposed [11].

In this paper, we shall discuss dynamics of atomatom entanglement in a superradiant Bose-Einstein condensate.

\section{ENTANGLEMENT AND QUANTUM PHASE TRANSITIONS IN THE DICKE MODEL}

In this section we present a quick review of quantum entanglement in the Dicke model in order to discuss the differences from superradiant Bose-Einstein condensate model. Dicke model is described by the model Hamiltonian

$$
H=\omega_{0} S_{z}+\omega a^{\dagger} a+\frac{g}{\sqrt{N}}\left(a^{\dagger}+a\right)\left(S_{+}+S_{-}\right),
$$

where single mode radiation field is described by boson operators $a$ while the atomic subsystem is described by collective pseudospin operators. As $N \longrightarrow \infty$, in the thermodynamic limit, at $g=\sqrt{\omega \omega_{0}} / 2$ the system undergoes a quantum phase transition to a superradiant phase from a normal phase. Superradiant phase is characterized by macroscopic number of excitations both in the field and in the atomic system while in the normal phase the excitations are low due to weak coupling. This spin-boson model can be transformed to a two-mode bosonic model in a quadratic form using the Holstein-Primakoff transformation [12] to replace spin operators with bosonic ones $b, b^{\dagger}$ so that the atom-field interaction $H_{a f}$ can be given of the form,

$$
H_{a f} \sim\left(a^{\dagger}+a\right)\left(b^{\dagger}+b\right) .
$$

From normal to superradiant phase the transition is identified to be a second order quantum phase tran- 
sition associated with the breaking of the global parity symmetry [13].

Characterization of entanglement with the entanglement entropy reveals that atom-field entanglement follows the critical behavior of quantum phase transition [9]. Effect of dissipation on the entanglement and quantum phase transitions is also discussed recently for some bosonic bath models [14].

Let us note that in the rotating wave approximation one would have $H_{a f} \approx\left(a^{\dagger} b+\right.$ h.c. $)$. Such a case has been considered in the first rigorous discussion of quantum phase transition in the Dicke model [15], which happens at $g=\sqrt{\omega \omega_{0}}$. Counter-rotating terms has significant effects however on quantum chaos [13].

\section{SUPERRADIANT BOSE-EINSTEIN CONDENSATE MODEL}

We shall now develop the Bose-Einstein superradiance model and show that under certain conditions it can be reduced to quadratic forms similar to Dicke Model with or without the rotating wave approximation that would accept analytical solutions.

We start with the same model used for the theory of superradiant scattering of laser light from Bose-Einstein condensate in Ref. [16]. A cigar shaped condensate, of length $L$ and width $W$, axially symmetric about the $z$ axis is considered. The incoming laser light with frequency $\omega_{0}$ is propagating along the $y$ axis, and its polarization is chosen along the $x$ axis. The incoming laser frequency $\omega_{0}$ is detuned from two-level atomic transition frequency $\omega_{a}$ by $\Delta=\omega_{0}-\omega_{a}$. Assuming faroff resonant laser light, adiabatic elimination of the excited state atomic field yields an effective Hamiltonian [16]

$$
\begin{aligned}
& \hat{H}=\int d^{3} \mathbf{r} \hat{\Psi}^{\dagger}(\mathbf{r}) \hat{H}_{0}(\mathbf{r}) \hat{\Psi}(\mathbf{r})+\int d^{3} \mathbf{k} \hbar \omega(\mathbf{k}) \hat{a}^{\dagger}(\mathbf{k}) \hat{a}(\mathbf{k}) \\
& +\int d^{3} \mathbf{k} d^{3} \mathbf{r}\left[\hbar g(\mathbf{k}) e^{i\left(\mathbf{k}_{0}-\mathbf{k}\right) \cdot \mathbf{r}} \hat{\Psi}^{\dagger}(\mathbf{r}) \hat{a}^{\dagger}(\mathbf{k}) \hat{\Psi}(\mathbf{r})+\text { h.c. }\right]
\end{aligned}
$$

where $\mathbf{k}_{0}=\left(\omega_{0} / c\right) \hat{\mathbf{y}}, \hat{\Psi}(\mathbf{r})$ is the ground state atomic field operator, and $\hat{a}(\mathbf{k})\left(\hat{a}^{\dagger}(\mathbf{k})\right)$ is the annihilation (creation) operator for a scattered photon with wave vector $\mathbf{k}$ in the frame rotating at $\omega_{0}$, and as such has frequency $\omega(\mathbf{k})=c|\mathbf{k}|-\omega_{0}$. Collisions between the ground state atoms, and the multiple scattering events between the scattered (vacuum) modes are neglected [16]. The coupling coefficient for the dominant process of incoming field and vacuum mode scattering is given by [16]

$$
g(\mathbf{k})=\frac{\left|\Omega_{0}\right|}{2 \Delta} \sqrt{\frac{c|\mathbf{k}| d^{2}}{2 \hbar \epsilon_{0}(2 \pi)^{3}}}|\hat{\mathbf{k}} \times \hat{\mathbf{x}}|,
$$

where $d$ is the magnitude of atomic dipole moment for the transition due to incoming laser interaction.
A quasimode (also called as atomic momentum sidemode) expansion of the atomic field operator is introduced in the form [16]

$$
\hat{\Psi}(\mathbf{r})=\sum_{\mathbf{q}}\langle\mathbf{r} \mid \mathbf{q}\rangle \hat{c}_{\mathbf{q}}(t)
$$

where $\langle\mathbf{q} \mid \mathbf{r}\rangle=\phi_{0}(\mathbf{r}) e^{i \mathbf{q} \cdot \mathbf{r}}$ and $\left[\hat{c}_{\mathbf{q}}, \hat{c}_{\mathbf{q}^{\prime}}^{\dagger}\right]=\left\langle\mathbf{q} \mid \mathbf{q}^{\prime}\right\rangle \cong \delta_{\mathbf{q}, \mathbf{q}^{\prime}}$. $\phi_{0}(\mathbf{r})$ and $\hbar \mu$ are the ground state and ground state energy of $H_{0}$, respectively. $\omega_{\mathbf{q}}=\hbar|\mathbf{q}|^{2} / 2 m$ is the sidemode energy due to recoil.

Substituting the expansion (5) into Eq. (3) reduces the effective Hamiltonian to the form

$$
\begin{aligned}
\hat{H}= & \int d^{3} \mathbf{k} \hbar \omega(\mathbf{k}) \hat{a}(\mathbf{k})^{\dagger} \hat{a}(\mathbf{k})+\sum_{\mathbf{q}} \hbar \omega_{\mathbf{q}} \hat{c}_{\mathbf{q}}^{\dagger} \hat{c}_{\mathbf{q}} \\
& +\sum_{\mathbf{q} \mathbf{q}^{\prime}} \int d^{3} \mathbf{k} g(\mathbf{k}) \rho_{\mathbf{q}, \mathbf{q}^{\prime}}(\mathbf{k}) \hat{c}_{\mathbf{q}}^{\dagger} \hat{a}_{\mathbf{k}}^{\dagger} \hat{a}_{\mathbf{k}_{0}} \hat{c}_{\mathbf{q}^{\prime}}
\end{aligned}
$$

in a frame rotating at frequency $\mu$. Here $\rho_{\mathbf{q}, \mathbf{q}^{\prime}}(\mathbf{k})=$ $\int d \mathbf{r}\left|\phi_{0}(\mathbf{r})\right|^{2} e^{i\left[(\mathbf{k}+\mathbf{q})-\left(\mathbf{k}_{0}+\mathbf{q}^{\prime}\right)\right] \cdot \mathbf{r}}$. For $L \gg W$ small angle Rayleigh scattering can be neglected. When the angular distribution about end-fire modes $\left(\mathbf{k}_{e}= \pm k_{0} \hat{z}\right)$ is sharply peaked Fresnel number becomes close to 1 [16]. Then, we consider the operator for the end-fire modes only. Furthermore, we only consider the contribution form the four sidemodes in the weak long incoming laser regime. The interaction Hamiltonian becomes

$$
\begin{gathered}
\hat{H}_{\mathrm{int}}=-\frac{N}{\Delta}|g|^{2}\left(\hat{c}_{+}^{\dagger} \hat{a}_{-}^{\dagger} \hat{a}_{0} \hat{c}_{0}+\hat{c}_{-}^{\dagger} \hat{a}_{+}^{\dagger} \hat{a}_{0} \hat{c}_{0}\right. \\
\left.+\hat{c}_{2}^{\dagger} \hat{a}_{-}^{\dagger} \hat{a}_{0} \hat{c}_{-}+\hat{c}_{2}^{\dagger} \hat{a}_{+}^{\dagger} \hat{a}_{0} \hat{c}_{+}\right)+ \text {h.c. },
\end{gathered}
$$

where $N=\int d^{3} \mathbf{r}\left|\phi_{0}(\mathbf{r})\right|^{2}=1$. Following short hand notations are used:

$$
\hat{a}_{ \pm} \equiv \hat{a}_{ \pm \mathbf{k}_{e}}, \quad \hat{a}_{0} \equiv \hat{a}_{\mathbf{k}_{0}}, \quad \hat{c}_{ \pm} \equiv \hat{c}_{\left(\mathbf{k}_{0} \pm \mathbf{k}_{e}\right)}, \quad \hat{c}_{2}=\hat{c}_{2 \mathbf{k}_{0}} .
$$

This atom-field interaction is rather different from the usual Dicke model. It contains four-mode interactions and it is not a quadratic form.

\section{DYNAMICAL EVOLUTION OF THE SYSTEM}

In order to examine entanglement properties of superradiant condensate model, we aim to reduce it into a quadratic form. For that purpose, we consider some dynamical regimes separately.

\section{Initial Times of Evolution of the System}

(1) When we are interested in the short-time dynamics, we may consider the condensate atoms with 
zero momentum $\left\langle n_{0}\right\rangle$ approximately constant. Then, we change the operator $\hat{c}_{0}$ with the constant value $\beta=$ $e^{i \theta_{\beta}}|\beta|=e^{i \theta_{\beta}} \sqrt{N}$.

(2) Furthermore, the number of laser photons is large compared to number of condensate atoms $\left(N_{0}=\right.$ $\left.2 \times 10^{8} \gg N=4 \times 10^{6}\right)$, the depletion in the pump photons is neglected, such that $a_{0}$ is replaced by $\alpha_{0}=$ $e^{i \theta_{\alpha}}\left|\alpha_{0}\right|=e^{i \theta_{\alpha}} \sqrt{N_{0}}$.

(3) For the initial times the occupancy of $\left|c_{2}\right\rangle$ state is negligible, because $\left|c_{ \pm}\right\rangle$states are not effectively occupied to give second order scattering. The terms containing $\hat{c}_{2}$ are neglected.

Then we are left with the Hamiltonian

$$
\begin{aligned}
\hat{H}_{1}= & -\frac{\sqrt{N_{0} N}}{\Delta}|g|^{2}\left[\left(e^{i \theta} \hat{a}_{+}^{\dagger} \hat{c}_{-}^{\dagger}+e^{-i \theta} \hat{a}_{+} \hat{c}_{-}\right)\right. \\
& \left.+\left(e^{i \theta} \hat{a}_{-}^{\dagger} \hat{c}_{+}^{\dagger}+e^{-i \theta} \hat{a}_{-} \hat{c}_{+}\right)\right] .
\end{aligned}
$$

This Hamiltonian is exactly solvable and solutions are in the form of two-mode vacuum squeezed states.Transformations $b_{1}=\frac{e^{-i \theta / 2}}{\sqrt{2}}\left(a_{+}+c_{-}\right), b_{2}=$ $\frac{e^{-i \theta / 2}}{\sqrt{2}}\left(a_{+}-c_{-}\right) \quad$ and $\quad b_{3}=\frac{e^{-i \theta / 2}}{\sqrt{2}}\left(a_{-}+c_{+}\right), \quad b_{4}=$ $\frac{e^{-i \theta / 2}}{\sqrt{2}}\left(a_{-}+c_{+}\right)$decouple the Hamiltonian into the form $H=-\frac{\hbar \chi_{1}}{2}\left[\left(b_{1}^{2}+b_{1}^{\dagger 2}\right)-\left(b_{2}^{2}+b_{2}^{\dagger 2}\right)+\left(b_{3}^{2}+b_{3}^{\dagger 2}\right)-\right.$ $\left.\left(b_{4}^{2}+b_{4}^{\dagger 2}\right)\right]$, composed of four decoupled terms, where $\hbar \chi_{1}=\frac{\sqrt{N_{0} N}}{\Delta}|g|^{2}$. The solution to $H=\eta\left(a^{2}+\right.$ $\left.a^{\dagger 2}\right)$ is vacuum squeezed state. Side-mode and end-fire mode operators are obtained as

$$
\begin{aligned}
& a_{ \pm}(t)=\cosh \left(\chi_{1} t\right) a_{ \pm}+i e^{i \theta} \sinh \left(\chi_{1} t\right) c_{\mp}^{\dagger}, \\
& c_{\mp}(t)=\cosh \left(\chi_{1} t\right) c_{\mp}+i e^{i \theta} \sinh \left(\chi_{1} t\right) a_{ \pm}^{\dagger} .
\end{aligned}
$$

\section{Late Times of Evolution of the System}

(1) The scattering of second side-modes is significant, when $\left|c_{+}\right\rangle$occupied close about $N / 2$. This time, then, occupancy of $\left|c_{0}\right\rangle$ is negligible. We neglect the first two terms in (7).

(2) We assume that $\left|c_{2}\right\rangle$ reached the steady state, that occupation of $\left|c_{2}\right\rangle$ does not change much. We change the $c_{2}$ operator by constant $c=e^{-i \phi_{c}}|c|$.
(3) Depletion in the laser photons can be neglected, again: $a_{0} \longrightarrow \alpha_{0}=e^{i \phi_{\alpha}}\left|\alpha_{0}\right|$.

(4) Initial states are given by the final states of $a_{ \pm}\left(t_{0}\right)$ and $c_{ \pm}\left(t_{0}\right)$ operators, which are time evolved up to $t=$ $t_{0}$, under the act of the first Hamiltonian (9).

Hamiltonian simplifies to

$$
\begin{aligned}
\hat{H}_{2}=- & \sqrt{N_{0}}|c| \frac{|g|^{2}}{\Delta}\left[\left(e^{i \phi} a_{-}^{\dagger} c_{-}+e^{-i \phi} a_{-} c_{-}^{\dagger}\right)\right. \\
& \left.+\left(e^{i \phi} a_{+}^{\dagger} c_{+}+e^{-i \phi} a_{+} c_{+}^{\dagger}\right)\right] .
\end{aligned}
$$

After second side-modes are significant, time evolution of the operators are dealt by Hamiltonian (12). Then, we turn off the Hamiltonian (9) after $t>t_{0}$ and turn on the Hamiltonian (12). The initial state of the second Hamiltonian are given by the solutions of the first Hamiltonian at $t=t_{0}$.

Solutions to Hamiltonian (12) are $\left(\tau=t-t_{0}\right)$

$$
\begin{aligned}
& a_{ \pm}(t)=\cos \left(\chi_{2} \tau\right) a_{ \pm}\left(t_{0}\right)+i e^{i \phi} \sin \left(\chi_{2} \tau\right) c_{ \pm}\left(t_{0}\right), \\
& c_{ \pm}(t)=\cos \left(\chi_{2} \tau\right) c_{ \pm}\left(t_{0}\right)+i e^{-i \phi} \sin \left(\chi_{2} \tau\right) a_{ \pm}\left(t_{0}\right),
\end{aligned}
$$

where $a_{ \pm}\left(t_{0}\right)$ and $c_{ \pm}\left(t_{0}\right)$ are given by equations (10), (11) and $\hbar \chi_{2}=\sqrt{N_{0}}|c| \frac{|g|^{2}}{\Delta}$.

\section{CONTINUOUS VARIABLE ENTANGLEMENT CRITERIA}

We shall use continuous variable entanglement measure which is defined $[17,18]$ for two continuous variable operators $\hat{x}_{1}$ and $\hat{x}_{2}$ such that they are disentangled (separable) if the EPR-like operators

$$
\hat{u}=|s| \hat{x}_{1}+\frac{1}{s} \hat{x}_{2}, \quad \hat{v}=|s| \hat{p}_{1}-\frac{1}{s} \hat{p}_{2}
$$

satisfies the inequality relation

$$
\left\langle(\Delta \hat{u})^{2}\right\rangle+\left\langle(\Delta \hat{v})^{2}\right\rangle \geq s^{2}+\frac{1}{s^{2}}
$$

with real $s$. Without additional separability requirement, $\hat{u}-\hat{v}$ satisfies

$$
\left\langle(\Delta \hat{u})^{2}\right\rangle+\left\langle(\Delta \hat{v})^{2}\right\rangle \geq\left|s^{2}-\frac{1}{s^{2}}\right| .
$$

If criterion (16) is violated, however, $\left|x_{1}\right\rangle$ and $\left|x_{2}\right\rangle$ states are inseparable that they are entangled.

A convenient way to test entanglement is to define the parameter

$$
\lambda=\left\langle(\Delta \hat{u})^{2}\right\rangle+\left\langle(\Delta \hat{v})^{2}\right\rangle-\left(s^{2}+\frac{1}{s^{2}}\right)
$$


and check if $\lambda$ gets negative, so that separability condition (16) is violated. $\lambda$ is calculated to be if

$$
\lambda=2\left(s^{2}\left\langle c_{+}^{\dagger} c_{+}\right\rangle+\frac{1}{s^{2}}\left\langle c_{-}^{\dagger} c_{-}\right\rangle-\left|\left\langle c_{+} c_{-}+c_{+}^{\dagger} c_{-}^{\dagger}\right\rangle\right|\right),
$$

where $c_{+}$and $c_{-}$are the annihilation operators and related to $\hat{x}_{1,2}$ and $\hat{p}_{1,2}$ as $\hat{x}_{1,2}=\left(c_{ \pm}+c_{ \pm}^{\dagger}\right) / \sqrt{2}$ and $\hat{p}_{1,2}=\left(c_{ \pm}-c_{ \pm}^{\dagger}\right) / i \sqrt{2} \cdot|s|$ is given by

$$
s^{2}=\sqrt{\left\langle c_{-}^{\dagger} c_{-}\right\rangle /\left\langle c_{+}^{\dagger} c_{+}\right\rangle} .
$$

However, equation (19) is valid when $\left\langle\hat{x}_{i}\right\rangle=0$ and $\left\langle\hat{p}_{i}\right\rangle=0$ or $\langle\hat{u}\rangle^{2}=\langle\hat{v}\rangle^{2}=0$ [19]. We need to generalize it to make it applicable for our condensate superradiance case in which nonzero values of $\langle\hat{u}\rangle,\langle\hat{v}\rangle$ could occur by

$$
\begin{aligned}
\lambda=2\left(s^{2}\left\langle c_{+}^{\dagger} c_{+}\right\rangle\right. & \left.+\frac{1}{s^{2}}\left\langle c_{-}^{\dagger} c_{-}\right\rangle-\left|\left\langle c_{+} c_{-}+c_{+}^{\dagger} c_{-}^{\dagger}\right\rangle\right|\right) \\
& -\langle\hat{u}\rangle^{2}-\langle\hat{v}\rangle^{2} .
\end{aligned}
$$

Expectation values are $\langle\hat{u}\rangle=\sqrt{2}|s| \alpha_{1}+\sqrt{2} \alpha_{2} / s$ and $\langle\hat{v}\rangle=\sqrt{2}|s| \beta_{1}-\sqrt{2} \beta_{2} / s$ where $\alpha_{1,2}=$ $\operatorname{Re}\left\{\left\langle c_{+,-}\right\rangle\right\} \beta_{1,2}=\operatorname{Im}\left\{\left\langle c_{+,-}\right\rangle\right\}$. Then, entanglement criteria can be written as

$$
\begin{gathered}
\lambda=2 s^{2}\left(\left\langle\hat{c}_{+}^{\dagger} \hat{c}_{+}\right\rangle-\left|\left\langle\hat{c}_{+}\right\rangle\right|^{2}\right)+2 \frac{1}{s}\left(\left\langle\hat{c}_{-}^{\dagger} \hat{c}_{-}\right\rangle-\left|\left\langle\hat{c}_{-}\right\rangle\right|^{2}\right) \\
-4 \frac{s}{|s|}\left(\operatorname{Re}\left(\left\langle\hat{c}_{+} \hat{c}_{-}\right\rangle\right)-\alpha_{1} \alpha_{2}+\beta_{1} \beta_{2}\right) .
\end{gathered}
$$

In this generalization $c$ parameter is redefined to be

$$
s^{2}=\sqrt{\frac{\left\langle\hat{c}_{-}^{\dagger} \hat{c}_{-}\right\rangle-\left|\left\langle\hat{c}_{-}\right\rangle\right|^{2}}{\left\langle\hat{c}_{+}^{\dagger} \hat{c}_{+}\right\rangle-\left|\left\langle\hat{c}_{+}\right\rangle\right|^{2}}},
$$

and the sign of $s$ is determined by $\operatorname{sgn}(s)=$ $\operatorname{sgn}\left(\operatorname{Re}\left\{\left\langle\hat{c}_{+} \hat{c}_{-}\right\rangle\right\}-\alpha_{1} \alpha_{2}+\beta_{1} \beta_{2}\right)$. In our further discussions we shall have the symmetry $\left\langle c_{+}\right\rangle=\left\langle c_{-}\right\rangle$so that have $s^{2}=1$, which means that minimum value of parameter is $\lambda=-2$ for our case.

\section{ATOM-ATOM ENTANGLEMENT}

We are now ready to investigate the entanglement between the side-mode atoms, $\left|c_{+}\right\rangle$and $\left|c_{-}\right\rangle$. The solutions are already given in equations $(10),(11)$ for earlier times and (13), (14) for later times.
Using these we calculate the time evolutions of the $\hat{c}_{ \pm}(t)$ operators as

$$
\begin{gathered}
\hat{c}_{+}(t)=C_{1} C_{2} \hat{c}_{-}+i e^{i \theta} S_{1} C_{2} \hat{a}_{+}^{\dagger}+i e e^{-i \phi} C_{1} S_{2} \hat{a}_{-} \\
-e^{i(\theta-\phi)} S_{1} S_{2} \hat{c}_{+}^{\dagger}, \\
\hat{c}_{-}(t)=C_{1} C_{2} \hat{c}_{+}+i e^{i \theta} S_{1} C_{2} \hat{a}_{-}^{\dagger}+i e^{-i \phi} C_{1} S_{2} \hat{a}_{+} \\
-e^{i(\theta-\phi)} S_{1} S_{2} \hat{c}_{-}^{\dagger},
\end{gathered}
$$

where $C_{1} \equiv \cosh \left(\chi_{1} t_{0}\right), S_{1} \equiv \sinh \left(\chi_{1} t_{0}\right)$ and $C_{2} \equiv$ $\cos \left(\chi_{2}\left(t-t_{0}\right)\right), S_{2} \equiv \sin \left(\chi_{2}\left(t-t_{0}\right)\right)$.

The entanglement parameter, between the sidemodes now reads out to be

$$
\begin{gathered}
\lambda_{s s}=s^{2}\left\langle\hat{N}_{-}(t)\right\rangle+\left\langle\hat{N}_{+}\right\rangle / s^{2} \\
-\frac{s}{|s|}\left\langle\hat{c}_{-}(t) \hat{c}_{+}(t)+\hat{c}_{-}^{\dagger}(t) \hat{c}_{+}^{\dagger}(t)\right\rangle .
\end{gathered}
$$

Then, we can express the behavior of the entanglement parameter more explicitly as follows

$$
\begin{gathered}
\lambda_{s s}(t)=2\left(2 \sinh ^{2}\left(\chi_{1} t_{0}\right)\right. \\
\left.-\cos (\theta+\phi)\left(\sinh \left(2 \chi_{1} t_{0}\right) \sin \left(2 \chi_{2}\left(t-t_{0}\right)\right)\right)\right) .
\end{gathered}
$$

In order to obtain entanglement we need to choose $\cos (\theta+\phi) \simeq 1$ as $2 \sinh ^{2}\left(\chi_{1} t_{0}\right) \sim N, \lambda(t) \sim 2 N$ at $t=t_{0}$. However, $\lambda_{s s}$ decreases continuously down to -2 . Since negative value of $\lambda$ implies the inseparability [18], the existence of entanglement is found. Although -2 seems small compared to the initial value of $\lambda \sim 2 N$, it is quite high when compared in the inequality (16). $\lambda=-2$ is the lowest value, due to (17), one would get for entanglement criteria parameter (since $s=1)$.

\section{CONCLUSIONS}

Quantum correlations in superradiant Bose-Einstein condensate atoms are discussed. A four mode boson model is developed to describe superradiant condensate. This model can be reduced to quadratic models similar to those considered for Dicke model superradiance in thermal equilibrium. Such reductions are argued to occur in the condensate dynamically. It is shown that atoms in the superradiant atomic condensate can exhibit Einstein-Podolsky-Rosen (EPR)-type quantum correlations during the evolution of the superradiant system and become continuous variable entangled.

\section{ACKNOWLEDGMENTS}

M.Ö.O. is supported by a TÜBA/GEBIP grant and TÜBITAK-KARIYYER grant no. 104T165. 


\section{REFERENCES}

1. S. Sachdev, Quantum Phase Transitions (Cambridge Univ. Press, Cambridge, 1999).

2. L. Amico, R. Fazio, A. Osterloh, and V. Vedral, Rev. Mod. Phys. 80, 517 (2008).

3. H. J. Lipkin, N. Meshkov, and A. J. Glick, Nucl. Phys. 62, 188 (1965); N. Meshkov, A. J. Glick, and H. J. Lipkin, Nucl. Phys. 62, 199 (1965); A. J. Glick, H. J. Lipkin, and N. Meshkov, Nucl. Phys. 62, 211 (1965).

4. R. Orús, S. Dusuel, and J. Vidal, e-print, condmat/08033151 (2008).

5. A. Osterloh, L. Amico, G. Falci, and R. Fazio, Nature (London) 416, 608 (2002).

6. T. J. Osborne and M. A. Nielsen, Phys. Rev. A 66, 032110 (2002).

7. G. Vidal, J. I. Latorre, E. Rico, and A. Kitaev, Phys. Rev. Lett. 90, 227902 (2003).

8. R. H. Dicke, Phys. Rev. 93, 99 (1954).

9. N. Lambert, C. Emary, and T. Brandes, Phys. Rev. Lett. 92, 073602 (2004).
10. S. Inouye, A. P. Chikkatur, D. M. Stamper-Kurn, J. Stenger, D. E. Pritchard, and W. Ketterle, Science 285, 571 (1999).

11. M. G. Moore and P. Meystre, Phys. Rev. Lett. 85, 5026 (2000).

12. T. Holstein and H. Primakoff, Phys. Rev. 58, 1098 (1958).

13. C. Emary and T. Brandes, Phys. Rev. E 67, 066203 (2003).

14. K. L. Hur, Ann. Phys. 323, 2208 (2008).

15. K. Hepp and E. Lieb, Ann. Phys. (New York) 76, 360 (1973); Y. K. Wang and F. T. Hioe, Phys. Rev. A 7, 831 (1973).

16. M. G. Moore and P. Meystre, Phys. Rev. Lett. 83, 5202 (1999).

17. Y. Ping, B. Zhang, Z. Cheng, and Y. Zhang, Phys. Lett. A 362, 128 (2007).

18. L. M. Duan, G. Giedke, J. I. Cirac, and P. Zoller, Phys. Rev. Lett. 84, 2722 (2000).

19. J. Xia and G. C. Guo, Chin. Phys. Lett. 21, 1877 (2004). 